\title{
Acessibilidade física urbana em campus universitário como requisito do direito à educação superior 1
}

\section{University campus urban physical accessibility as prerequisite of higher education right}

\section{Kalil, Rosa Maria Locatelli 1; Gelpi, Adriana 2; Oliveira, Wagner Mazetto de 3; Corrêa, Matheus Behnen 4 ; Pinto, Fábio Lamaison 5 ; Pasa, Ritiéli 6; Gayeski, Morgana Terezinha ${ }^{7}$}

\author{
1 Universidade de Passo Fundo, Rodovia BR-285, km 292, Passo Fundo, RS, \\ Brasil, kalil@upf.br \\ 2 Universidade de Passo Fundo, agelpi@upf.br \\ 3 Universidade Federal do Rio Grande do Sul wagnermazzetto@gmail.com \\ 4 Universidade de Passo Fundo, 165643@upf.br \\ 5 Universidade de Passo Fundo, 144428@upf.br \\ 6 Universidade de Passo Fundo, 170697@upf.br \\ 7 Universidade de Passo Fundo, morganagayeski31@gmail.com
}

\begin{abstract}
RESUMO
A possibilidade de incluir os estudantes no ensino superior tem como barreira a falta de acessibilidade física nos espaços universitários. A adequação de campi universitários tem sido indicada tanto em diretrizes educacionais internacionais quanto nas brasileiras. A par disso, as normas técnicas dispõem de padrões de acessibilidade para áreas externas e internas. Este trabalho tem como objetivo apresentar os resultados de proposta de adequação do Campus Universitário da Universidade de Passo Fundo, que sofreu intervenção nos espaços urbanos. Após diagnósticos, projetos e intervenções, foi realizada a avaliação pós-ocupação, por meio de questionários on-line. Os resultados demonstram bom grau de conhecimento e interesse sobre acessibilidade pelos usuários da UPF. Enfatizam as questões de acessibilidade e sua importância na garantia de frequência e permanência no ensino superior de pessoas com deficiência.
\end{abstract}

Palavras-chave: acessibilidade universal, campus universitário, direito à educação superior.

\section{ABSTRACT}

The possibility of including students in higher education has as a barrier the lack of physical accessibility in university spaces. The adequacy of university campuses has been indicated in both international and Brazilian educational guidelines. In addition, the technical standards have accessibility standards for external and internal areas. This paper aims to present the

\footnotetext{
${ }^{1}$ KALIL, Rosa Maria Locatelli; GELPI, Adriana; OLIVEIRA, Wagner Mazetto de; CORRÊA, Matheus Behnen; PINTO, Fábio Lamaison; PASA, Ritiéli; GAYESKI, Morgana Terezinha. Acessibilidade em campus universitário como requisito do direito à educação superior. In: SIMPÓSIO NACIONAL DE GESTÃO E ENGENHARIA URBANA: SINGEURB, 2, 2019, São Paulo. Anais [...]. Porto Alegre: ANTAC, 2019.
} 
results of a proposed adaptation of the University Campus of the University of Passo Fundo, which has undergone intervention in urban spaces. After diagnosis, projects and interventions, the post-occupation evaluation was carried out through an online questionnaire. The results indicate that the results demonstrate a good degree of knowledge and interest about accessibility by UPF users. They emphasize that accessibility issues and their importance in ensuring attendance and permanence in higher education of people with disabilities.

Keywords: universal accessibility, university campus, right to higher education.

\section{INTRODUÇÃO}

A educação como direito universal das pessoas é valorizada desde a Carta das Nações Unidas (UN, 1945) e consolidou-se pela Convenção Internacional dos Direitos Econômicos, Sociais e Culturais (UN, 1966). No Brasil, houve incorporação de políticas de acessibilidade na Constituição Federal de 1988, pois "a legislação deve determinar normas de construção de espaços públicos e edificações de uso público e fabricação de veículos de transporte público, de forma a garantir acesso adequado para pessoas com deficiência" (BRASIL, 1988). Em 2000, as leis 10.048 e 10.098 estabeleceram tratamento diferenciado, normas gerais e critérios básicos para a promoção da acessibilidade autônoma para pessoas com deficiência em edificações, espaços urbanos, mobiliário urbano e equipamentos. Essas legislações foram regulamentadas pelo Decreto Federal 5.296 de dezembro de 2004 (BRASIL, 2004), com diretrizes para adaptação de edificações às normas técnicas de acessibilidade da NBR 9050 2004 (ABNT, 2004).

Em 2008, a Política Nacional para Educação Especial na Perspectiva da Educação Inclusiva estabeleceu o propósito de inclusão desde os primeiros anos até a educação superior. Esta legislação seguiu diretrizes da Convenção das Nações Unidas para os Direitos das Pessoas com Deficiência (UN, 2007), incorporada como emenda constitucional no Brasil pelo Decreto $n^{\circ}$ 6.949/2009 (BRASIL, 2009).

A consolidação dos direitos ocorre com o Estatuto das Pessoas com Deficiência, que objetiva assegurar os direitos, promover oportunidades iguais, dar autonomia e garantir acessibilidade. O documento afirma que educação constitui direito de pessoas com deficiência, assegurando um sistema educacional inclusivo em todos os níveis e ao longo da vida. Assegura acesso à educação superior, profissional e tecnológica, educação em oportunidades e condições iguais a outras pessoas (BRASIL, 2015).

Este trabalho objetiva apresentar a percepção dos usuários da UPF acerca da implantação de rotas acessíveis nas áreas urbanas do campus I. Utilizou-se como metodologia a avaliação pós-ocupação (APO), com inquérito on-line aos usuários.

\section{AVALIAÇÃO PÓS-OCUPAÇÃO E ACESSIBILIDADE EM CAMPUS UNIVERSITÁRIOS}

Os campus universitários são espaços privilegiados para a educação superior, servindo como laboratórios vivos para o desenvolvimento sustentável e experiências múltiplas. Conforme o documento da Década para a Educação Sustentável, a educação superior tem um papel específico importante para desempenhar. As universidades devem funcionar como lugares de pesquisa e aprendizagem para o desenvolvimento sustentável e como polos de inovação e atividades para suas comunidades e nacionalmente (UN, 2005). É responsabilidade social das lideranças e da comunidade acadêmica prover locais adequados para todos as pessoas para atender diversas atividades de ensino e pesquisa em instituições de educação superior (UN, 2015). Os requisitos de acessibilidade física são estabelecidos para construção e operação dos ambientes universitários novos e existentes pelo Ministério da Educação (BRASIL, 2004; BRASIL 2009) e pelos governos estaduais e municipais. 


\subsection{A APO e a acessibilidade na educação superior brasileira}

A avaliação pós-ocupação demonstra ser uma ferramenta útil para a melhoria dos espaços para a educação superior. Com respeito à acessibilidade física, por incluir os usuários como fontes de referência, colabora para uma avaliação efetiva que pode resultar em significativos melhoramentos em estabelecimentos educacionais e campus universitários.

Visto que a acessibilidade física é um pré-requisito para a entrada e permanência de estudantes, a contribuição desta metodologia tem sido enfatizada em várias instituições. Estudos sobre barreiras no campus da Universidade Federal do Rio de Janeiro (UFRJ) revelaram a necessidade de acessibilidade universal (DUARTE; COHEN, 2004). O Campus de Bauru da Universidade de São Paulo foi mapeado para intervenções realizadas com base na ABNT (LAMÔNICA et al., 2008; NAOE, 2013). Na Universidade Federal da Paraíba foi desenvolvido o projeto "UFPB para todos: eliminando barreiras". organizando rota acessível no Campus I com projeto piloto para estabelecimento de diretrizes mestras (COSTA et al., 2012). Com base na experiência de estudante cadeirante foram identificadas barreiras arquitetônicas na Universidade Federal do Pará (UFPA), concluindo que o atendimento às normas de acessibilidade torna possível a inclusão na educação superior (COSTA; SOUZA, 2014).

\subsection{Acessibilidade Universal no Campus da UPF}

Na Universidade de Passo Fundo (UPF), a preocupação com as rotas urbanas acessíveis iniciou-se em 2014, com a adequação dos espaços do campus às novas demandas do Estatuto da Cidade e das diretrizes do MEC. O Laboratório de Estudos Urbanos e Regionais (Laburb) desenvolveu projeto de adaptação dos espaços públicos e edificações para a acessibilidade.

O campus central da UPF é uma referência educacional na região, atraindo veículos de muitos municípios do norte do estado, automóveis, ônibus, bicicletas e veículos de carga. Devido às suas características, recebe cerca de 14.000 estudantes e profissionais, dentre os quais 26 pessoas com deficiência (visual, auditiva e cadeirantes). Por isso, tornou-se necessária a adaptação dos espaços urbanos, com vistas à democratização dos espaços públicos e do próprio ensino superior.

\section{PROCEDIMENTOS METODOLÓGICOS}

O projeto piloto de acessibilidade universal no Campus I da UPF foi projetado como parte da mobilidade urbana sustentável do campus. Buscou implantar rotas acessíveis, incluindo a adequação de vias e espaços públicos, adequação de passeios e acessos aos edifícios e adaptando o sistema de transporte aos princípios de acessibilidade universal, conforme Figura 1 . 


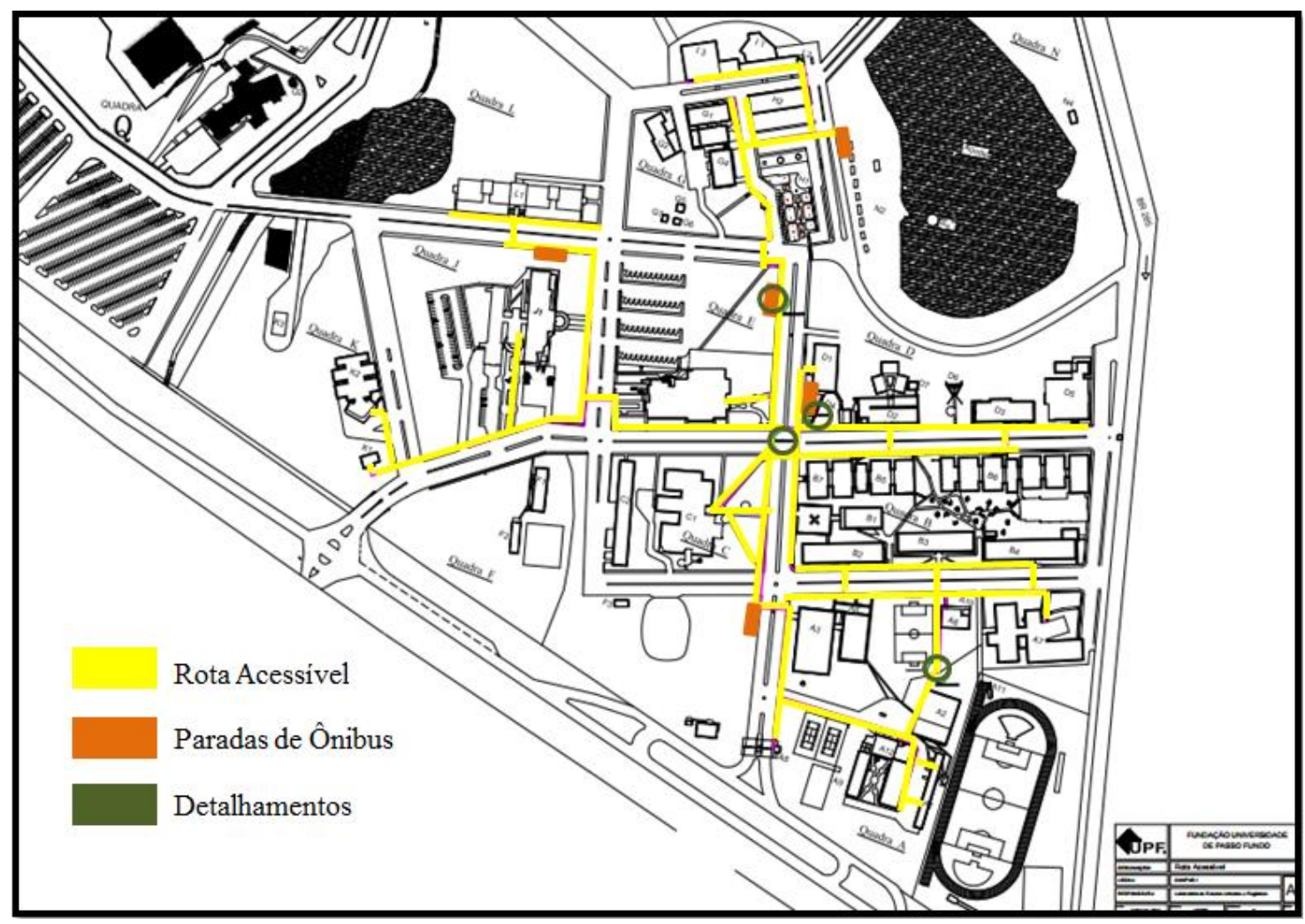

Fonte: Os autores

As etapas metodológicas incluíram: 1) Revisão bibliográfica de padrões de acessibilidade universal, legislação; 2) Estudo de casos de espaços acessíveis em campi universitários e áreas urbanas; 3) Diagnóstico de acessibilidade no Campus I da UPF; 4) Elaboração de desenho urbano e detalhamento de infraestruturas acessíveis para rotas, passeios, rampas, paradas de ônibus e travessias; 5) Pesquisa de materiais de construção para adaptar o campus conforme os padrões projetados; 6) Trabalho conjunto com a equipe de construção para adaptar os padrões às condições físicas, estruturais, técnicas e financeiras; 7) Avaliação pós-ocupação das rotas adaptadas, etapa apresentada neste trabalho.

\section{RESULTADOS E DISCUSSÃO DA AVALIAÇÃO PÓS-OCUPAÇÃO}

A aplicação dos questionários foi realizada on-line, em outubro de 2017, disponível para todos os estudantes, professores e funcionários. O instrumento foi composto de doze questões, sobre as características dos respondentes e suas opiniões e conhecimento sobre acessibilidade e rotas acessíveis e adaptações do campus.

O questionário foi respondido por 234 pessoas, 63,3\% mulheres e 36,7 \% homens. Dos respondentes, $17,5 \%$ reportaram pessoas com deficiência em sua família e $29,4 \%$ em suas classes, demonstrando que o interesse em respondente possa ter sido motivado pela convivência com essas pessoas. Sobre o local de origem ou moradia dos respondentes, 72,65\% reside em Passo Fundo, 6,84\% em Carazinha, 3,42\% em Marau, 2, $14 \%$ em Lagoa Vermelha, 2,14\% em Tapejara e nos demais em municípios da região.

Quanto à faixa etária, pessoas entre 20 e 24 anos $(24,79 \%)$ predominaram, o que é característica dos estudantes universitários, e respondentes de mais de 30 anos foram mais da metade dos respondentes (Tabela 1). 
Tabela 1 - Faixa etária dos respondentes dos questionários

\begin{tabular}{ccc}
\hline Faixa etária & \multicolumn{2}{c}{ Respondentes } \\
\hline 17 a 19 anos & $13,68 \%$ & 32 \\
20 a 24 anos & $24,79 \%$ & 58 \\
25 a 29 anos & $11,54 \%$ & 27 \\
30 a 34 anos & $13,25 \%$ & 31 \\
35 a 39 anos & $10,68 \%$ & 25 \\
40 a 44 anos & $8,55 \%$ & 20 \\
45 a 49 anos & $8,12 \%$ & 19 \\
Mais de 50 anos & $9,40 \%$ & 22 \\
\hline Total & & 234 \\
\hline
\end{tabular}

Fonte: Os autores

Em relação à escolaridade, a maioria dos respondentes são alunos de graduação $(45,30 \%)$, seguidos dos respondentes com mestrado (13,25\%) (Tabela 2).

Tabela 2 - Escolaridade dos respondentes do questionário

\begin{tabular}{ccc}
\hline Níveis de escolaridade & \multicolumn{2}{c}{ Respondentes } \\
\hline Ensino médio completo & $1,28 \%$ & 3 \\
Cursando graduação & $45,30 \%$ & 106 \\
Graduação & $8,97 \%$ & 21 \\
Especialização/MBA em andamento & $4,70 \%$ & 11 \\
Especialização/MBA & $11,97 \%$ & 28 \\
Mestrado em andamento & $3,85 \%$ & 9 \\
Mestrado & $13,25 \%$ & 31 \\
Doutorado em andamento & $2,99 \%$ & 7 \\
Doutorado completo & $7,69 \%$ & 18 \\
\hline Total & & 234 \\
\hline
\end{tabular}

Fonte: Os autores

Acerca do conhecimento sobre acessibilidade e sua relação com cidadania e infraestrutura, as respostas foram organizadas em uma escala tipo Lickert de 1 a 5 , sendo - $1=$ desconheço e $5=$ conheço bastante sobre o tema. As médias das respostas das questões se concentraram acima de 3,5, indicando que os respondentes são conhecedores da questão da acessibilidade (Tabela 3). Contudo, muitos ainda desconhecem este tema, mesmo apresentando bom grau de escolarização e a maioria frequentando o ensino superior (Tabela 2) 
Tabela 3 - Níveis de conhecimento sobre acessibilidade

\begin{tabular}{|c|c|c|c|c|c|c|c|c|c|c|c|c|}
\hline & 1 & & 2 & & 3 & & 4 & & 5 & & Total & Média \\
\hline $\begin{array}{l}\text { Você sabe } \circ \text { que significa } \\
\text { acessibilidade? }\end{array}$ & $5,13 \%$ & 12 & $14,53 \%$ & 34 & $28,21 \%$ & 66 & $29,91 \%$ & 70 & $22,22 \%$ & 52 & 234 & 3,5 \\
\hline $\begin{array}{l}\text { Você sabe o que significa ser uma } \\
\text { pessoa com deficiência (PcD)? }\end{array}$ & $3,42 \%$ & 8 & $5,13 \%$ & 12 & $12,82 \%$ & 30 & $32,05 \%$ & 75 & $46,58 \%$ & 109 & 234 & 4,13 \\
\hline $\begin{array}{l}\text { Você sabe que democracia e } \\
\text { cidadania são ligados à } \\
\text { acessibilidade urbana? }\end{array}$ & $3,42 \%$ & 8 & $6,84 \%$ & 16 & $21,37 \%$ & 50 & $25,21 \%$ & 59 & $43,16 \%$ & 101 & 234 & 3,98 \\
\hline $\begin{array}{lll}\text { Você sabe } & \text { que para ter } \\
\text { acessibilidade } & \text { universal, } & \text { há }\end{array}$ & & & & & & & & & & & & \\
\hline $\begin{array}{l}\text { necessidade de } \\
\text { adequada } \\
\text { travessias, rampas)? }\end{array}$ & $1,71 \%$ & 4 & $3,85 \%$ & 9 & $5,13 \%$ & 12 & $28,21 \%$ & 66 & $61,11 \%$ & 143 & 234 & 4,43 \\
\hline $\begin{array}{l}\text { Você sabe o que é um piso } \\
\text { podotátil? }\end{array}$ & $11,54 \%$ & 27 & $6,84 \%$ & 16 & $10,68 \%$ & 25 & $23,08 \%$ & 54 & $47,86 \%$ & 112 & 234 & 3,89 \\
\hline
\end{tabular}

Fonte: Os autores

Para o nível de qualificação da rota acessível implementada no Campus, as respostas concentraram-se em Bom, com a média entre 3 e 5 em todas as questões (Tabela 4), que. indica um grau de qualificação com tendência positiva. A soma de respostas Bom e Muito Bom foi mais alta do que $55 \%$ em todos os itens avaliados. Esses resultados demonstram o acerto na implementação, mas denotam a necessidade de qualificar a existente e de ampliar para outros espaços do Campus ainda não acessíveis.

Tabela 4 - Níveis de qualificação da rota acessível implantada no Campus da UPF

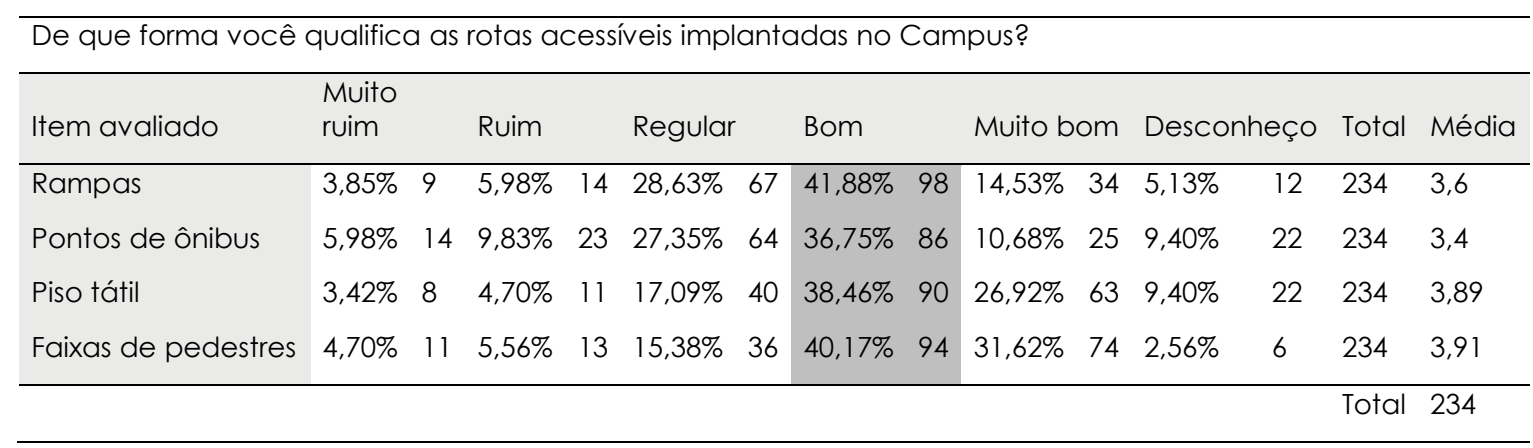

Fonte: Os autores

\section{CONSIDERAÇÕES FINAIS}

Os resultados demonstram bom grau de conhecimento e interesse sobre acessibilidade pelos usuários do campus I da UPF. O questionário será repetido em anos seguintes, de forma online e presencial e outras técnicas de APO serão aplicadas na pesquisa. Pretende servir como fator de educação para a acessibilidade e a sustentabilidade, ao mesmo tempo em que alimenta a complementação do projeto para garantia de inclusão.

Embora os esforços para obtenção de acessibilidade física urbana esteja determinado na legislação, e em fase de implementação nas instituições de ensino superior brasileiras, ainda é muito restrita sua abrangência. A atratividade de pessoas com deficiência para a educação superior brasileira requer investimento na infraestrutura física para tornar cotidiana sua presença nas atividades de ensino, pesquisa e extensão, contribuindo para a responsabilidade social universitária na formação e no desenvolvimento. 


\section{REFERÊNCIAS}

ABNT-ASSOCIAÇÃO BRASILEIRA DE NORMAS TÉCNICAS. NBR 9050. Acessibilidade de pessoas portadoras de deficiências a edificações, espaço, mobiliário e equipamento urbanos: procedimentos. Rio de Janeiro: ABNT, 2004.

BRASIL. Constituição da República Federativa do Brasil. Brasília: Imprensa Oficial, 1988. Disponível em: http://www.planalto.gov.br/ccivil_03/constituicao/constituicao.html. Acesso em: 02 ago. 2015.

BRASIL. Decreto Legislativo ${ }^{\circ}$ 5296. Brasília: Senado Federal, 2004.

http://www.planalto.gov.br/ccivil_03/_ato2004-2006/2004/decreto/d5296.htm. Acesso em: 30 jan. 2018.

BRASIL. Decreto legislativo $n^{\circ}$ 6949. Brasília: Senado Federal, 2009. Disponível em: http://www.planalto.gov.br/ccivil_03/_ato2007-2010/2009/decreto/d6949.htm Acesso em: 09 nov. 2015.

BRASIL. Lei Brasileira de Inclusão da Pessoa com Deficiência (Estatuto da Pessoa com Deficiência). Brasília, 2015. Disponível em: http://www.planalto.gov.br/cci -vil_03/_Ato20152018/2015/Lei/L13146.htm. Acesso em: 09 nov. 2015.

COSTA, A. L.; COURA, P. V.; GOMES, M. A.; PEREGRINA, Y. R.; SARMENTO, B. R.; SOUSA, R. A. Ergonomics issues in conceiving an accessible project. Work, Amsterdam, n. 41, p. 1403-1408, 2012. DOI: 10.3233/WOR-2012-0331-1403 IOS Press.

COSTA, M. F.; SOUZA, C. T. Acessibilidade e inclusão de cadeirantes na Universidade Federal do Pará. Rev. Ibero-Americana de Estudos em Educação, Araraquara, v.9, n.2, 2012.

DUARTE, C. R.; COHEN, R. Acessibilidade aos espaços do ensino e pesquisa: desenho universal na UFRJ - possivel ou utópico? In: SEMINÁRIO INTERNACIONAL NUTAU 2004: DEMANDAS SOCIAIS, INOVAÇÕES TECNOLÓGICAS E A CIDADE. Anais eletrônicos... São Paulo: Universidade de São Paulo, 2004.

LAMÔNICA, D. A.; ARAÚJO FILHO, P.; SIMOMELLI, S. B.; CAETANO, V. L.; REGINA, M. R.; REGIANI, D. M. Accessibility in the university environment: identification ofarchitectural barriers in the USP Campus of Bauru. Rev. Bra. Ed. Esp. , Bauru, v.14, n. 2, maio-ago. 2008, p. 177-188.

NAOE, A. USP Legal ajuda a superar barreiras impostas às pessoas com deficiência. USP Online Destaque, 2013. Disponível em: http://www5.usp.br/37627/usp-legal-ajuda-a-superarbarreiras-impostas-as-pessoas-com-deficiencia/. Acesso em: 28 jan. 2018.

UN-UNITED NATION. Charter of the United Nations. New York: General Assembly, 1945.

UN-UNITED NATIONS. Convention on the Rights of Persons with Disabilities. 2007. Disponivel em: http://www.un.org/disabilities/documents/convention/convention_accessible_pdf.pdf. Acesso em: 28 jan. 2018.

UN-UNITED NATIONS. International Convenant on Economic, Social and Cultural Rights. NeW York NY 16 December 1966: United Nations General Assembly. 
UN-UNITED NATIONS. Transforming our world: the 2030 Agenda for Sustainable Development. (2015). Disponível em:

http://www.un.org/ga/search/view_doc.asp?symbol=A/RES/70/1 \&Lang=E. Acesso em: 25 jan. 2018.

UN-UNITED NATIONS. United Nations Decade of Education for Sustainable Development. (2005). Disponível em: http://daccess- dds-

ny.un.org/doc/UNDOC/GEN/N04/490/48/PDF/N0449048.pdf? OpenElement. Acesso em: 27 jan. 2018. 